\title{
Hot Topics in Clinical Oral Implants Research: Recent Trends in Literature Coverage
}

\author{
Vesela Valkova ${ }^{1}$, Ceeneena Ubaidha Maheen ${ }^{1}$, Bernhard Pommer ${ }^{2, *}$, Xiaohui Rausch-Fan ${ }^{3}$ \\ and Rudolf Seemann ${ }^{4}$ \\ 1 Medical University of Vienna, Bernhard Gottlieb School of Dentistry, Sensengasse 2a, \\ A-1090 Vienna, Austria; vesela.e.valkova@gmail.com (V.V.); ceeneena@yahoo.com (C.U.M.) \\ 2 Academy for Oral Implantology, Lazarettgasse 19/DG, A-1090 Vienna, Austria \\ 3 Medical University of Vienna, Rausch-Fan Laboratory, Sensengasse 2a, A-1090 Vienna, Austria; \\ xiaohui.rausch-fan@meduniwien.ac.at \\ 4 Medical University of Vienna, Department of Oral and Maxillofacial Surgery, Währinger Gürtel 18-20, \\ A-1090 Vienna, Austria; rudolf.seemann@meduniwien.ac.at \\ * Correspondence: pommer@implantatakademie.at; Tel.: +43-1-4028668; Fax: +43-1-402-866-810 \\ Academic Editor: Patrick Schmidlin \\ Received: 6 January 2016; Accepted: 18 May 2016; Published: 24 May 2016
}

\begin{abstract}
This systematic review looks at thematic trends in clinical research publications on dental implants. For this purpose, MEDLINE electronic searches as well as additional hand searches of six main journals in the field were conducted. A total of 2875 clinical studies published between 2001 and 2012 met the inclusion criteria and were subjected to statistical analysis. Hot topics in dental implant literature included immediate loading $(14.3 \%)$, bone substitutes $(11.6 \%)$, cross-arch full bridges $(8.0 \%)$, and immediate implant placement $(7.5 \%)$. A significant increase in scientific interest for immediate loading $(+6.3 \%, p=0.001)$, platform switching $(+2.9 \%, p=0.001)$, guided implant surgery $(+1.9 \%, p=0.011)$, growth factors $(p=0.014,+1.4 \%)$, piezoelectric surgery $(+1.3 \%, p=0.015)$, and restorative materials $(+0.7 \%, p=0.011)$ was found. A declining scientific interest in onlay grafting $(-0.3 \%, p=0.042)$ was recorded. The findings regarding current clinical oral implants research tie in with better-informed consumers and increased patient demands. Our results demonstrate an increasing interest in techniques that avoid complicated procedures such as bone grafting and that reduce treatment duration.
\end{abstract}

Keywords: immediate implant loading; guided implant surgery; minimally invasive techniques; immediate implant placement

\section{Introduction}

The present special issue of Dentistry Journal deals with "Advances in Implant Dentistry," and the following keywords denote hot topics in this field: template-guided implant placement, minimally invasive techniques, short lengths and reduced implant diameters, novel bone grafting techniques, medically compromised patients, peri-implantitis treatment, immediate placement and restoration, transition from a failing dentition, CAD/CAM prosthetics, and optical intraoral impressions. As the first paper in this special issue, the following review aims to provide the background to recent trends and "hot topics" in advanced and minimally invasive oral implant treatment [1].

The concept of osseointegration of oral implants was introduced by Branemark 40 years ago and set the precedent for new knowledge in oral medicine. Since then, oral implantology has become one of the most investigated topics in dental medicine, with exponential growth in the use of implant products [2]. Data shows that the number of implants used for oral rehabilitation in the USA increased ten-fold between 1983 and 2002 and also ten-fold from 2000 to 2010 [3]. While previously the primary 
aim of research on oral implantology was to find ways to rehabilitate function [4], many efforts nowadays are focused on the shortening of treatment procedures, simplifying surgical techniques, and esthetic improvement [5]. It is well known that oral implantology is a prosthetically driven field with a major surgical component [6]. Therefore, the current state of the art in implant dentistry represents advances in both surgical and prosthodontic techniques [5].

Keeping pace with research development, the aim of this systematic review was to investigate contemporary issues in oral implantology research and to perform a topical trend analysis of clinical studies published in the time period from 2001 to 2012.

\section{Material and Methods}

\subsection{Search Strategy}

A MEDLINE electronic literature search was conducted, limited to clinical studies on dental implants published between 2001 and 2012. The search term "dental implant," sorted by "year of publication" was used in order to capture all relevant articles [7]. Additional hand searching was performed to examine six main journals in the field: The International Journal of Maxillofacial Implants, Journal of Oral Implantology, Clinical Oral Implant Related Research, Implant Dentistry, European Journal of Oral Implantology, and Clinical Oral Implant Research. Two reviewers independently identified all trials [8]. The PubMed search initially identified 15,695 publications, and 5048 additional results were identified by hand search. These studies were screened for their relevance based upon a threshold set [9]:

- inclusion criteria: prospective and retrospective studies, cross-sectional studies, case-control studies, case reports with at least 10 patients

- exclusion criteria: non-English publications, statistical studies, animal studies, finite element analyses, in vitro studies, review articles, and case series with fewer than 10 patients.

A total number of 3695 articles were subjected to abstract review. Where the abstract provided little information, a full text analysis was performed. Authors of potentially relevant publications, which were not available or lacked data, were contacted and asked for cooperation. Ultimately, 2875 clinical studies were identified as meeting the inclusion criteria. Our goal was to investigate how trends change over time as regards the topics examined in modern implant dentistry research. In this respect, we have determined that 31 topics were appropriate: 23 of them concerned surgical issues and 8 dealt with prosthodontic issues (Table 1). First, all relevant publications were screened for the topics listed in Table 1 independently by two reviewers. Thereafter, the results were verified, and all doubtful publications were discussed before the final decision was taken. 
Table 1. Topics sorted by literature coverage. Absolute numbers of publications per year as well as the total percentage of all clinical papers $2001-2012{ }^{*}$ indicates prosthodontic topics).

\begin{tabular}{|c|c|c|c|c|c|c|c|c|c|c|c|c|c|}
\hline Topic & 2001 & 2002 & 2003 & 2004 & 2005 & 2006 & 2007 & 2008 & 2009 & 2010 & 2011 & 2012 & Total \\
\hline Immediate loading * & 10 & 7 & 21 & 19 & 26 & 27 & 42 & 50 & 36 & 40 & 52 & 81 & $14.3 \%$ \\
\hline Bone substitutes & 13 & 18 & 20 & 16 & 24 & 20 & 15 & 30 & 33 & 44 & 53 & 46 & $11.6 \%$ \\
\hline Cross-arch implant bridges * & 13 & 10 & 24 & 20 & 16 & 11 & 11 & 22 & 20 & 20 & 25 & 39 & $8.0 \%$ \\
\hline Immediate implant placement & 7 & 10 & 9 & 13 & 7 & 7 & 23 & 21 & 21 & 30 & 30 & 38 & $7.5 \%$ \\
\hline Simultaneous implant placement with augmentation & 11 & 12 & 10 & 18 & 10 & 10 & 8 & 16 & 21 & 21 & 19 & 28 & $6.4 \%$ \\
\hline Implant design & 9 & 8 & 8 & 12 & 4 & 10 & 17 & 9 & 17 & 10 & 12 & 18 & $4.7 \%$ \\
\hline Early loading * & 6 & 8 & 12 & 9 & 14 & 8 & 13 & 17 & 6 & 14 & 8 & 14 & $4.5 \%$ \\
\hline Onlay grafting & 5 & 7 & 7 & 7 & 12 & 11 & 9 & 12 & 14 & 8 & 20 & 11 & $4.3 \%$ \\
\hline Medically compr. patients & 5 & 7 & 7 & 4 & 14 & 9 & 9 & 12 & 9 & 12 & 13 & 15 & $4.0 \%$ \\
\hline Healing modality & 6 & 10 & 9 & 7 & 11 & 8 & 8 & 14 & 10 & 9 & 7 & 7 & $3.7 \%$ \\
\hline Transcrestal sinus floor elevation & 2 & 5 & 2 & 3 & 7 & 5 & 5 & 9 & 10 & 10 & 11 & 18 & $3.0 \%$ \\
\hline Implant diameter & 4 & 2 & 3 & 8 & 2 & 6 & 6 & 6 & 7 & 10 & 6 & 17 & $2.7 \%$ \\
\hline Flapless surgery & 0 & 1 & 1 & 2 & 2 & 4 & 12 & 17 & 2 & 12 & 10 & 14 & $2.7 \%$ \\
\hline Socket grafting & 1 & 2 & 5 & 4 & 4 & 2 & 3 & 7 & 10 & 10 & 12 & 15 & $2.6 \%$ \\
\hline Guided surgery & 0 & 0 & 3 & 3 & 2 & 4 & 9 & 7 & 5 & 12 & 5 & 17 & $2.3 \%$ \\
\hline Implant FPD-s & 8 & 2 & 5 & 5 & 3 & 5 & 8 & 5 & 3 & 5 & 6 & 11 & $2.3 \%$ \\
\hline Implant number & 1 & 1 & 4 & 1 & 3 & 3 & 3 & 3 & 5 & 10 & 15 & 17 & $2.3 \%$ \\
\hline Growth factors & 2 & 0 & 4 & 2 & 6 & 2 & 5 & 7 & 12 & 9 & 7 & 8 & $2.2 \%$ \\
\hline Implant length & 2 & 0 & 2 & 5 & 7 & 4 & 5 & 5 & 4 & 6 & 6 & 15 & $2.1 \%$ \\
\hline Peri-implantitis therapy & 3 & 2 & 0 & 2 & 3 & 7 & 7 & 2 & 6 & 2 & 9 & 8 & $1.8 \%$ \\
\hline Platform switching * & 1 & 0 & 0 & 0 & 0 & 3 & 2 & 2 & 12 & 10 & 10 & 10 & $1.7 \%$ \\
\hline Restorative materials * & 0 & 3 & 1 & 2 & 2 & 4 & 2 & 6 & 3 & 4 & 6 & 10 & $1.5 \%$ \\
\hline Tilted implants & 1 & 0 & 0 & 0 & 3 & 0 & 4 & 3 & 3 & 7 & 8 & 10 & $1.4 \%$ \\
\hline Abutment design * & 4 & 1 & 3 & 2 & 0 & 1 & 4 & 2 & 5 & 3 & 10 & 3 & $1.3 \%$ \\
\hline Smoking & 0 & 3 & 1 & 4 & 2 & 3 & 2 & 3 & 3 & 2 & 4 & 4 & $1.1 \%$ \\
\hline Piezoelectric surgery & 0 & 0 & 0 & 0 & 1 & 0 & 2 & 4 & 1 & 8 & 2 & 7 & $0.9 \%$ \\
\hline Early implant placement & 0 & 1 & 0 & 2 & 2 & 1 & 0 & 3 & 3 & 1 & 2 & 4 & $0.7 \%$ \\
\hline Cantiliver FPD-s * & 0 & 0 & 1 & 3 & 0 & 2 & 1 & 1 & 1 & 0 & 2 & 4 & $0.5 \%$ \\
\hline Cement vs. Screw retention * & 0 & 0 & 0 & 2 & 0 & 3 & 0 & 2 & 1 & 0 & 2 & 3 & $0.5 \%$ \\
\hline Ceramic implants & 0 & 0 & 0 & 0 & 0 & 1 & 1 & 0 & 1 & 2 & 1 & 4 & $0.4 \%$ \\
\hline Pterygoid implants & 0 & 0 & 1 & 0 & 1 & 0 & 1 & 0 & 1 & 0 & 0 & 1 & $0.2 \%$ \\
\hline
\end{tabular}




\subsection{Statistical Analysis}

As mentioned above, 2875 publications were analyzed. In order to find statistical trends in respect to the relevant topics between 2001 and 2012, Poisson regression analysis was performed, taking the level of significance as $p \leqslant 0.05$, using R-project statistical software version 3.1.0. This statistical test was used to model count data, which in this case was the number of publications. $p$-values were calculated for every topic, taking into account the relative number publications per topic from the total number of publications.

\section{Results}

The surgical and prosthodontic topics of interest were computed as percentages of the total number of publications (Table 1). Among the most covered surgical topics in the literature were immediate loading $(14.3 \%)$, bone substitutes $(11.6 \%)$, immediate implant placement $(7.5 \%)$, simultaneous implant placement with bone augmentation $(6.4 \%)$, onlay grafting $(4.3 \%)$, medically compromised patients $(4.0 \%)$, healing modality $(3.7 \%)$, transcrestal sinus floor elevation $(3.0 \%)$, flapless surgery $(2.7 \%)$, socket grafting $(2.6 \%)$, and guided surgery $(2.4 \%)$. Immediate loading $(14.3 \%)$, cross-arch implant bridges $(8.0 \%)$, early loading $(4.5 \%)$, and platform switching $(1.7 \%)$ were ranked as the most prevalent prosthodontic issues in current oral implant research.

The surgical issues were the more prevalent topics, demonstrating an increasing rate of publications over the time in terms of mean coverage $(0.53 \pm 0.01)$ per publication (Figure 1$)$, as compared to prosthodontic issues $(0.33 \pm 0.05$ hits). The mean coverage values were estimated based on yearly ratios: the number of prosthodontic/surgical publications per year in relation to the total number of publications per year. The significant increase in publications on surgical issues over the years was demonstrated by Poisson regression analysis $(p=0.002)$.

A total of eight topics showed significant trends $(p<0.05)$ over the years 2001 to 2012 (Table 2). Immediate loading demonstrated the highest increase with a positive change of $+6.3 \%$ and $p=0.001$ (Figure 2a). Platform switching $(+2.9 \%, p=0.001)$ was the second topic showing a significant increase; however, only one relevant article was detected between 2001 and 2006 (Figure 2b). These topics were followed by guided implant surgery $(+1.9 \%, p=0.011)$, growth factors $(+1.4 \%, p=0.014)$, piezoelectric surgery $(+1.3 \%, p=0.015)$, and restorative materials $(+0.7 \%, p=0.011)$. The green line represents the percentage of the total number of publications for every year. The black trend line reveals the relationship between the year of publication ( $x$-variable) and the percentage of the total number of publications (y-variable). Since there were no publications on platform switching between 2002 and 2005, there is a negative trend line intercept starting from 2001 (Figure 2b). Decreasing scientific interest and a corresponding downward trend were recorded for the topic onlay grafting $(-0.3 \%, p=0.042)$.

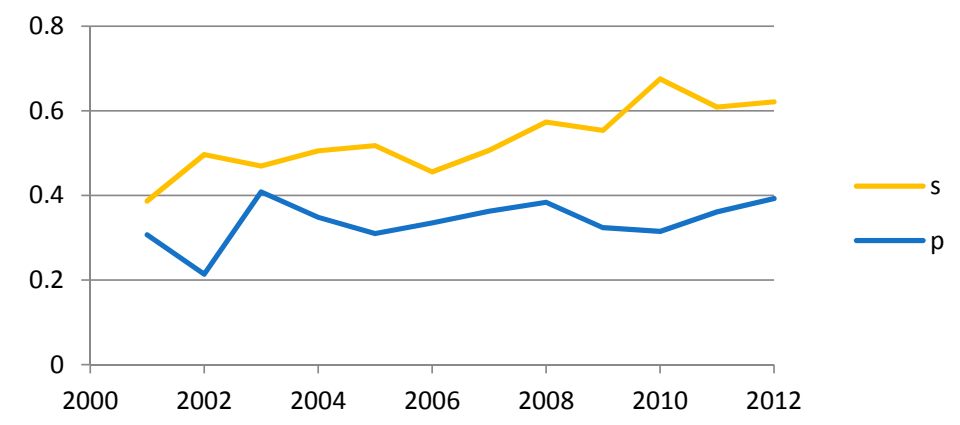

Figure 1. Literature coverage of surgical (s) versus prosthodontic (p) issues: $x$-axis indicates year of publication, $\mathrm{y}$-axis indicates the ratio of numbers of publications (surgical/prosthodontic) to the total number of publications per year. 
Table 2. Topics demonstrating a significant increase (positive) or decrease (negative change) of scientific interest in the years 2001-2012.

\begin{tabular}{cccccc}
\hline Topic & $\mathbf{2 0 0 1 - 2 0 0 4}$ & $\mathbf{2 0 0 5 - 2 0 0 8}$ & $\mathbf{2 0 0 9 - 2 0 1 2}$ & $p$-value & Change \\
\hline Immediate loading & $57(9.1 \%)$ & $145(16.2 \%)$ & $209(15.4 \%)$ & 0.001 & $+6.3 \%$ \\
Platform switching & $1(0.2 \%)$ & $7(0.8 \%)$ & $42(3.1 \%)$ & 0.001 & $+2.9 \%$ \\
Flapless implant surgery & $4(0.6 \%)$ & $35(3.9 \%)$ & $38(2.8 \%)$ & 0.001 & $+2.2 \%$ \\
Guided implant surgery & $6(1.0 \%)$ & $22(2.5 \%)$ & $39(2.9 \%)$ & 0.011 & $+1.9 \%$ \\
Growth factors & $8(1.3 \%)$ & $20(2.2 \%)$ & $36(2.7 \%)$ & 0.014 & $+1.4 \%$ \\
Piezoelectric surgery & $0(0.0 \%)$ & $7(0.8 \%)$ & $18(1.3 \%)$ & 0.015 & $+1.3 \%$ \\
Restorative materials & $6(1.0 \%)$ & $14(1.6 \%)$ & $23(1.7 \%)$ & 0.011 & $+0.7 \%$ \\
Onlay grafting & $26(4.2 \%)$ & $44(4.9 \%)$ & $53(3.9 \%)$ & 0.042 & $-0.3 \%$ \\
\hline
\end{tabular}

\section{Discussion}

Comparisons of published clinical trials per year revealed a trend of increasing interest in conducting clinical trials, starting with 137 relevant articles in the year 2001 and reaching the number of 446 publications in the year 2012. However, even the total number of 3695 articles is smaller than the total number of 4655 clinical studies published between 1989 and 1999 reported by Russo et al. [10]. Given that the number of publications increased with every year, it was considered more appropriate to perform Poisson regression analysis related to percentage-based values rather than related to absolute values for all topics.

Immediate loading proved to be the most studied topic in the last decade (Figure 2a). This avid scientific interest can be explained by several advantages it offers, such as shortened treatment protocols, immediate rehabilitation of the function, and high patient satisfaction. Meta-analyses on single-tooth implant placement have shown encouraging results for the immediate loading protocol as a promising alternative to conventional loading, as it may be equally successful and may not significantly affect marginal bone resorption and implant success rates [11-13]. Another meta-analysis by Papaspyridakos et al. [14] reported that there was no significant difference between immediate, early, and conventional loading in edentulous patients with fixed prostheses, and all three protocols showed a high level of success. However, other reviewers disagree with this assessment of the unimpaired success of the immediate loading protocol. A meta-analysis of clinical studies comparing the immediate and conventional loading of single tooth implants discovered that immediate loading has a significantly higher risk of implant failure [15]. Schimmel and coworkers [16] concluded that, despite the high implant survival rates, the conventional and early loading protocols are superior to immediate loading as better documented protocols, providing better results in the first year of loading. A survey among implantologists from 16 countries all over the world stated that immediate loading was the treatment protocol most accepted by dentists in Australia and Europe [17]. Based on these controversial statements in the literature, it can be concluded that there is still a lack of well-designed RCTs concerning loading protocol [18] and immediate loading may well retain its place as a hot topic of discussion over the coming years.

The platform-switching concept arose in 1980 with the introduction of the wide diameter implants. Due to the lack of commercially available matching components for wide diameter implants, the standard-diameter abutments were used. Later, it was found that "platform-switched" implants demonstrated osseointegration with less initial crestal bone loss and were thus superior to the "platform-matched implants" [19]. However, the first introduction of this concept appeared in 2005 [20]. Radiographic observation over a period of 13 years demonstrated that platform switching resulted in little or no crestal bone loss as compared to the conventional implants, whereas marginal bone resorption of $1.5 \mathrm{~mm}$ on average was accepted as one of the criteria for success of the dental implant [21]. Our study shows that the increasing publication rate of clinical studies happened to coincide with the first official introduction of this concept, with a positive linear trend for this topic starting in 2005 (Figure 2b). Since guided surgery is performed in combination with the flapless procedure in most cases, [22] the similarity in literature coverage, illustrated in both scatter plots, does not come as a surprise (Figure 2c,d). 
In contrast to the last decade of the 20th century, when the main progress in the field of oral implant research was made in alveolar bone resorption management to refine the different graft techniques [23], our findings show that in the 21st century there has been increasing interest in methods developed to overcome the grafting procedures and even a loss of interest in one of the most used augmentation techniques, i.e., onlay bone grafting. It seems that dental implant scientific work is inspired more by the patient's appraisals [24], seeking to improve minimally invasive surgical techniques [25], diminish patient morbidity, and shorten the treatment time. However, the role of industrial funding for conducting clinical studies should be taken into consideration. $32.4 \%$ of the clinical trials are supported by industry as a source of funding, which is a suitable way for companies not only to comply with safety and efficacy standards, but also to introduce their new products to the market [26]. This industry sponsorship may lead to biased reporting and pro-industry conclusions [27]. This does have the potential to reflect on ongoing trends in clinical research. In this connection for instance, the relatively innovative technique of guided implant surgery provides less painful and invasive treatment but at the same time is a more difficult and expensive procedure than conventional implant placement, demonstrating the same survival rate. However, a survey by Hof and coworkers [28] showed that the main priority for the patients when it comes to implant therapy remains the predictability of treatment success. The achievements brought about by ongoing clinical research, such as improved quality, ease of use of implant systems, as well as shorter treatment duration [29] may provide grounds for future researchers to face the challenge of preserving the perspectives of clinical implant research, and specifically, to enhance the relationship between private practice and science without involving marketing.

In order to adhere to ethical rules on explicit reporting, including also the disadvantages of any study, the researchers are obligated to report their study's limitations. Undoubtedly, meta-analysis is the "gold standard" for performing any systematic review aiming at assessing treatment effects. Given that the variable investigated in the present study was the number of publications, the Poisson regression was selected as a statistical tool. The Poisson regression is used to model count data (in the present case this is the number of publications) and is an appropriate statistical method for predicting trends. Therefore, no methods estimating risk of bias, quality design, or heterogeneity of the studies provided by the meta-analysis were applied in this study.

A further limitation is presented by the use of only one database source. The findings in the present work are based on analysis, including studies from MEDLINE, and an additional hand search of six journals. However, the search strategy did not consider other databases such as EMBASE and the Cochrane Central Register of Controlled Trials.

In conclusion, the analysis of scientific literature on dental implants revealed several hot topics in the time period between 2001 and 2012. The most frequently covered surgical issues were bone substitutes $(11.6 \%)$ and immediate implant placement, $(7.5 \%)$, while the most prevalent prosthodontic topics involved immediate loading (14.3\%) and cross-arch full bridges (8.0\%). Given that the topics demonstrating the highest increase in interest were prosthodontic topics, i.e., immediate loading $(+6.3 \%)$ and platform switching $(+2.9 \%)$, the interest in researching prosthodontic topics will most likely continue to increase.

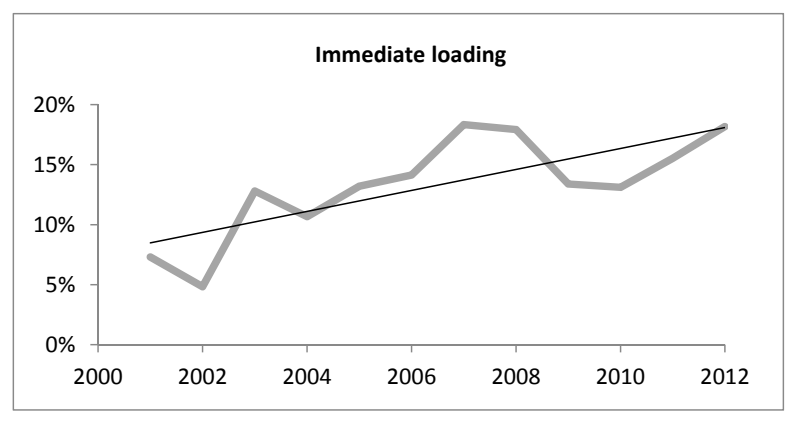

(a)

Figure 2. Cont. 


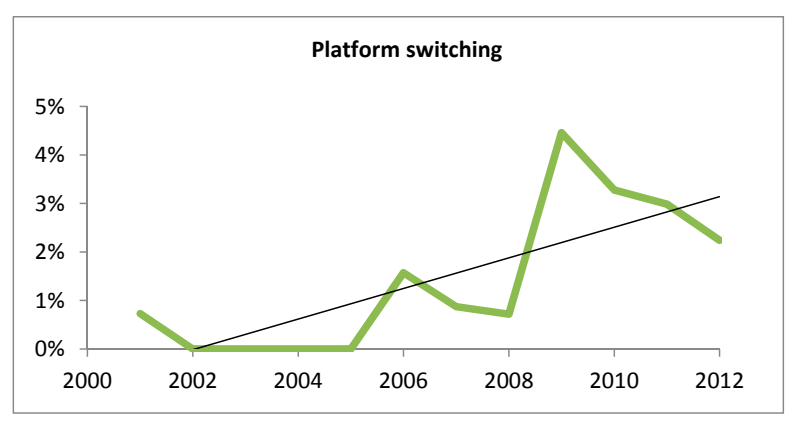

(b)

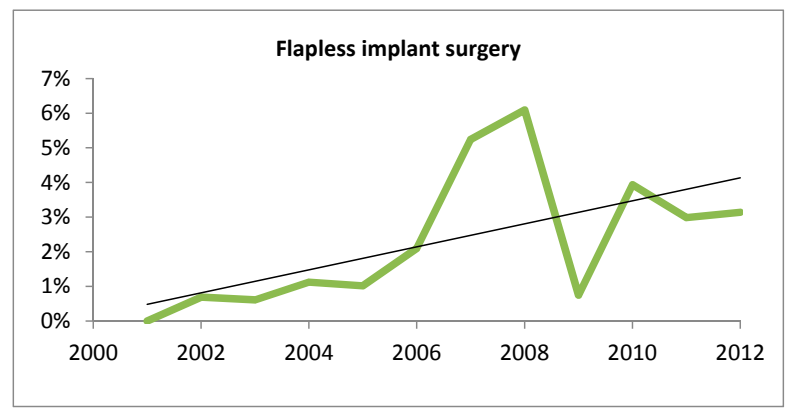

(c)

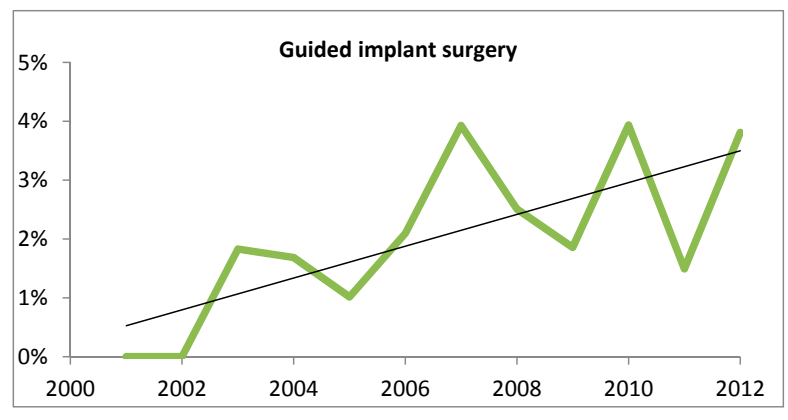

(d)

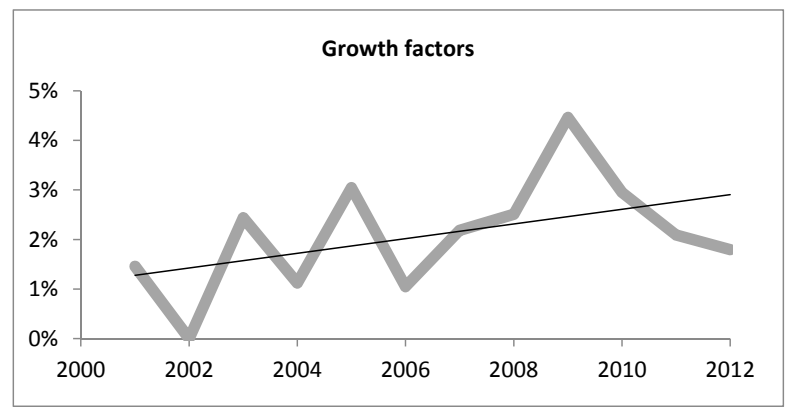

(e)

Figure 2. Cont. 


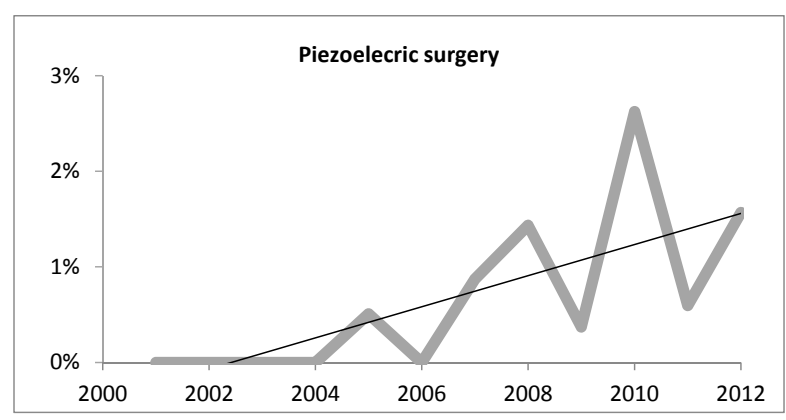

(f)

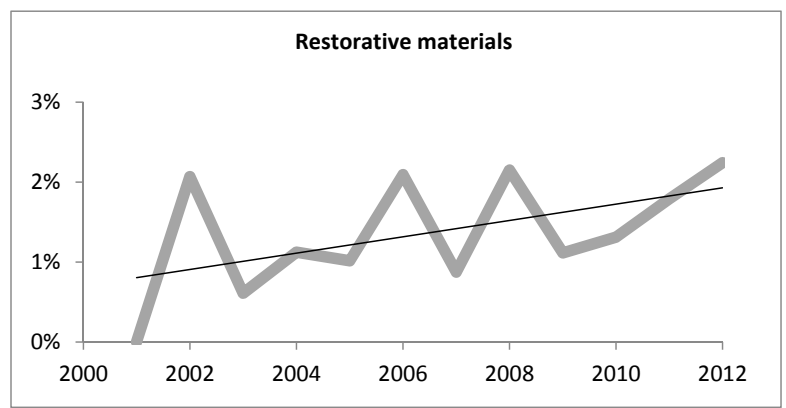

(g)

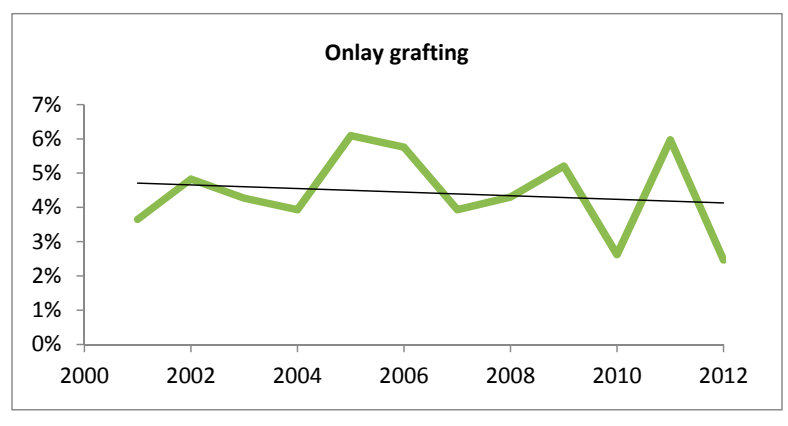

(h)

Figure 2. Trend curves (percentage out of the total number of publications per year) for (a) immediate loading; (b) platform switching; (c) flapless implant surgery; (d) guided implant surgery; (e) growth factors; (f) piezoelectric surgery; (g)restorative materials; and (h) onlay grafting.

Acknowledgments: No funding was received to support the study.

Author Contributions: Bernhard Pommer conceived and designed the experiments; Vesela Valkova and Ceeneena Ubaidha Maheen performed the experiments; Rudolf Seemann analyzed the data; Xiaohui Rausch-Fan contributed analysis tools; Vesela Valkova wrote the paper.

Conflicts of Interest: The authors declare no conflict of interest.

\section{References}

1. Heo, Y.Y.; Heo, S.Y.; Chang, M.W.; Park, J.M. The patient's satisfaction following implant treatment. J. Korean Acad. Prosthodont. 2008, 46, 569-576. [CrossRef]

2. Esposito, M.; Worthigton, H.V.; Coulthard, P. In search of truth: The role of systematic reviews and meta-analyses for assessing the effectiveness of rehabilitation with oral implants. Clin. Implant Dent. Relat. Res. 2001, 3, 62-78. [CrossRef] [PubMed] 
3. Misch, C. Rationale for implant dentistry. In Dental Implant Prosthetics, 2nd ed.; Misch, C., Ed.; Elsevier Mosby: St. Louis, MI, USA, 2014; Volume 1, p. 1.

4. Turkyilmaz, I.; Nicoll, R. State-of-the-Art Technology in Implant Dentistry: CAD/CAM, Implant Dentistry-A Rapidly Evolving Practice; Turkyilmaz, I., Ed.; ISBN: 978-953-307-658-4. InTech: Rijeka, Croatia. Available online: http://www.intechopen.com/books/implant-dentistry-a-rapidly-evolving-practice/stateof-the-art-technology-in-implant-dentistry-cad-cam (accessed on 14 December 2015). [CrossRef]

5. Pommer, B. Advances in implant dentistry-A special issue of dentistry Journal. Dent. J. 2015, 3, 132. [CrossRef]

6. Parkash, H. Computer aided implantology: Changing trends. Indian J. Dent. Res. 2015, 26, $223-224$. [CrossRef] [PubMed]

7. Linden, G.J.; Lyonos, A.; Scannapieco, F.A. Periodontal systematic associations: Review of the evidence. J. Periodontol. 2013, 84, 8-19. [CrossRef] [PubMed]

8. Van Tulder, M.; Furlan, A.; Bombardier, C.; Bouter, L. Editorial Board of the Cochrane Collaboration back review group; Updated method guidelines for systematic reviews in the Cochrane collaboration back review group. Spine (Phila Pa 1976) 2003, 28, 1290-1299. [CrossRef] [PubMed]

9. Kwok, V.; Caton, J.G.; Polson, A.M.; Hunter, P.G. Application of evidence-based dentistry: From research to clinical periodontal practice. Periodontol 2000 2012, 59, 61-74. [CrossRef] [PubMed]

10. Russo, S.P.; Fiorellini, J.P.; Weber, H.P.; Niedermann, R. Benchmarking the dental implant evidence on MEDLINE. Int. J. Maxillofac. Implants 2000, 15, 792-800.

11. Moraschini, V.; Porto Barboza, E. Immediate versus conventional loaded single implants in the posterior mandible: A meta-analysis of randomized controlled trials. Int. J. Oral Maxillofac. Surg. 2015. [CrossRef] [PubMed]

12. Benic, G.; Mir-Mari, J.; Hämerle, C.H. Loading protocols for single-implant crowns: A systematic review and meta analysis. Int. J. Oral Maxillofac. Implants 2014, 29, 222-238. [CrossRef] [PubMed]

13. Atieh, M.A.; Payne, A.G.; Duncan, W.J.; de Silva, R.K.; Cullinan, M.P. Immediate placement or immediate restoration/loading of single implants for molar tooth replacement: A systematic review and meta-analysis. Int. J. Oral Maxillofac. Implants 2010, 25, 401-415. [PubMed]

14. Papaspyridakos, P.; Mokti, M.; Chen, C.J.; Benic, G.I.; Galluci, G.O.; Chronopoulos, V. Implant and prosthodontic survival rates with implant fixed complete dental prostheses in the edentulous mandible after at least 5 years: A systematic review. Clin. Implant Dent. Rel. Res. 2014, 16, 705-717. [CrossRef] [PubMed]

15. Atieh, M.A.; Payne, A.G.; Duncan, W.J.; Cullinan, M.P. Immediate restoration/loading of immediately placed single implants: Is it an effective bimodal approach? Clin. Oral Implants Res. 2009, 20, 645-659. [CrossRef] [PubMed]

16. Schimmel, M.; Srinivasan, M.; Herrmann, F.R.; Müller, F. Loading protocols for implant-supported overdentures in the edentulous jaw: A systematic review and meta-analysis. Int. J. Oral Maxillofac. Implants 2014, 29 (Suppl. 1), 271-286. [CrossRef] [PubMed]

17. Chowdhary, R.; Hosadettu, S.R.; Chandrakar, N. A survey of the use of techniques, materials in dental implantology practice. Indian J. Dent. Res. 2014, 23, 297. [CrossRef] [PubMed]

18. Esposito, M.; Grusovin, M.G.; Maghaireh, H.; Worthington, H.V. Interventions for replacing missing teeth: Different times for loading dental implants. Cochrane Data Base Syst. Rev. 2013, 28, CD003878.

19. Kalavathy, N.; Sridevi, J.; Gehlot, R.; Kumar, S. "Platform switching": Serendipity. Indian J. Dent. Res. 2014, 25, 254-259. [CrossRef] [PubMed]

20. Baumgarten, H.; Cocchetto, R.; Testori, T.; Meltzer, A.; Porter, S. A new implant design for crestal bone preservation: Initial observations and case report. Pract. Proced. Aesthet. Dent. 2005, 17, 735-740. [PubMed]

21. Lazzara, R.J.; Porter, S.S. Platform switching: A new concept in implant dentistry for controlling postoperative crestal bone levels. Int. J. Periodontics Restor. Dent. 2006, 26, 9-17.

22. Browaeys, H.; Dierens, M.; Ruyffelaert, C.; Matthijs, C.; De Bruyn, H.; Vandeweghe, S. Ongoing crestal bone around implants subjected to computer-guided flapless surgery and immediate loading using all-on-4 concept. Clin. Implant Dent. Rel. Res. 2015, 17, 831-843. [CrossRef] [PubMed]

23. Laskin, D.M. Implantology 1997-Do we have all the answers? J. Oral Maxillofac. Surg. 1997, 55, 1371. [CrossRef]

24. Anderson, J.D. The need for criteria on reporting treatment outcomes. J. Prosthet. Dent. 1998, 79, 49-55. [CrossRef] 
25. Pommer, B.; Frantal, S.; Willer, J.; Posch, M.; Watzek, G.; Tepper, G. Impact of dental implant length on early failure rates: A meta-analysis of observational studies. J. Clin. Periodontol. 2011, 38, 856-863. [CrossRef] [PubMed]

26. Barao, V.A.; Shyamsunder, N.; Yuan, J.C.; Knoernshild, K.L.; Assuncao, W.G. Trends of funding, internatinalisation, and types of study for original articles published in five implant-related journals between 2005 and 2009. Int. J. Oral Maxillofac. Implants 2012, 27, 69-76. [PubMed]

27. Bekelman, J.E.; Liy, Y.; Gross, C.P. Scope and impact of financial conflicts of interest in biomedical research; A systematic review. JAMA 2003, 289, 454-465. [CrossRef] [PubMed]

28. Hof, M.; Tepper, G.; Semo, B.; Arnhart, C.; Watzek, G.; Pommer, B. Patient's perspectives on dental implant and bone graft surgery: Questionnaire-based interview survey. Clin. Oral Implants Res. 2014, 25, 42-45. [CrossRef] [PubMed]

29. Levin, R.; Keneth, J. Trends in Implant Dentistry. Dental Economics; 100(12). Available online: http://www. dentaleconomics.com/articles/print/volume-100/issue-12/features/trends-in-implant-dentistry.html (accessed on 16 December 2015).

(C) 2016 by the authors; licensee MDPI, Basel, Switzerland. This article is an open access article distributed under the terms and conditions of the Creative Commons Attribution (CC-BY) license (http://creativecommons.org/licenses/by/4.0/). 